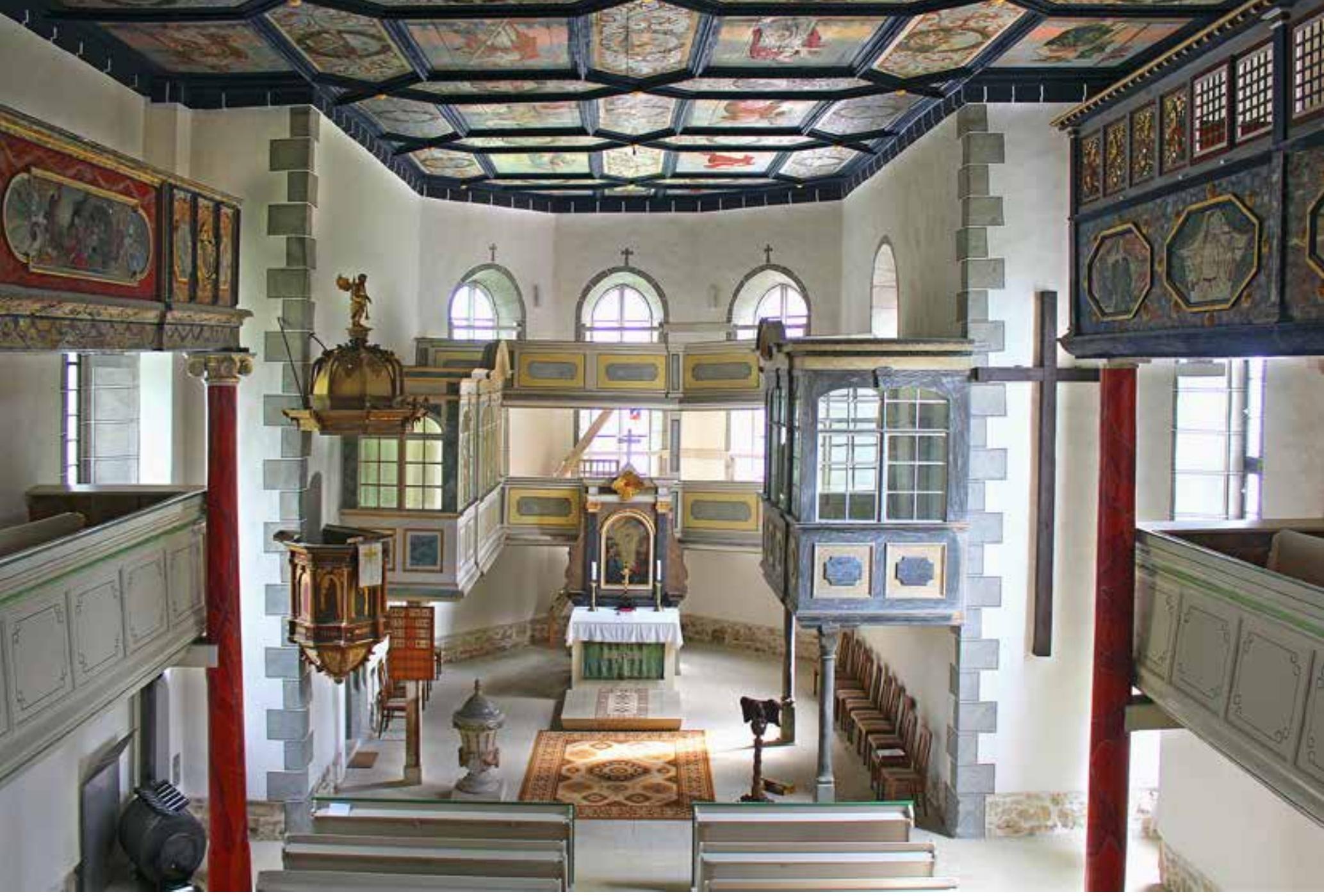

\title{
Die Embleme der Bilderdecke in der Kirche St. Gotthard in Jahna
}

\author{
Matthias Donath
}

Dass die Lommatzscher Pflege einmal eine sehr reiche Landschaft gewesen war, zeigt die Pfarrkirche St. Gotthard in Jahna (Gemeinde Ostrau, Landkreis Mittelsachsen). Der „Bauerndom“ ist über und über mit Einbauten gefüllt. Emporen drängeln sich um das Kirchenschiff; sie umgeben sogar den Chor. Die Einbauten folgen keiner einheitlichen Gestaltung, sondern sind $\mathrm{zu}$ unterschiedlichen Zeiten entstanden. Es handelt sich um Stiftungen der Bauern, die zum Gottesdienst in ihren eigenen Betstübchen oder auf ihren Kirchenstühlen sitzen wollten. Zu diesen hölzernen Einbauten treten die farbigen Bilderwelten christlicher Verkündigung. In diesem Aufsatz soll auf die bemalte Felderdecke hingewiesen werden, die bislang noch nie näher behandelt wurde, aber ein höchst spannendes Bildprogramm enthält.
Die flache hölzerne Decke wurde - laut Inschrift - 1679 von Johann Simon Lucas bemalt. ${ }^{1}$ Sie gliedert sich in 23 große rechteckige Bildfelder und 44 runde Medaillons. In den großen Bildfeldern wurden Jesus Christus als Heiland der Welt (Salvator Mundi), 13 Apostel und neun Propheten des Alten Testaments abgebildet. Die großen Bildfelder sind durch rautenförmige Rahmenfelder voneinander getrennt, die mit Ranken bemalt sind und zudem jeweils ein rundes Medaillon enthalten. Jedes Medaillon ist von einem grünen, mit vier Rosen geschmückten Kranz umgeben. Im oberen Teil des Bildfelds befindet sich jeweils ein weißes Schriftband mit schwarzer Beschriftung in Renaissance-Majuskeln.

Aus dem Gegenüber von Text und Bild ist zu erkennen, dass es sich hier um Embleme handelt. Diese Kunstgattung erfreute sich in der Frühen
St. Gotthard in Jahna, Innenansicht nach Osten, Zustand 2017 nach der Restaurierung Foto: Matthias Donath

1 Signatur im Feld mit der Darstellung des Propheten Habakuk: „Johann: Sim. Lucas / Pictor A(nn)o 1679.“

2 Hartmut Freytag/Wolfgang Harms/Michael Schilling: Gesprächskultur des Barock. Die Embleme der Bunten Kammer im Herrenhaus Ludwigsdorf bei Eckernförde. Kiel 2001, S. 8. 

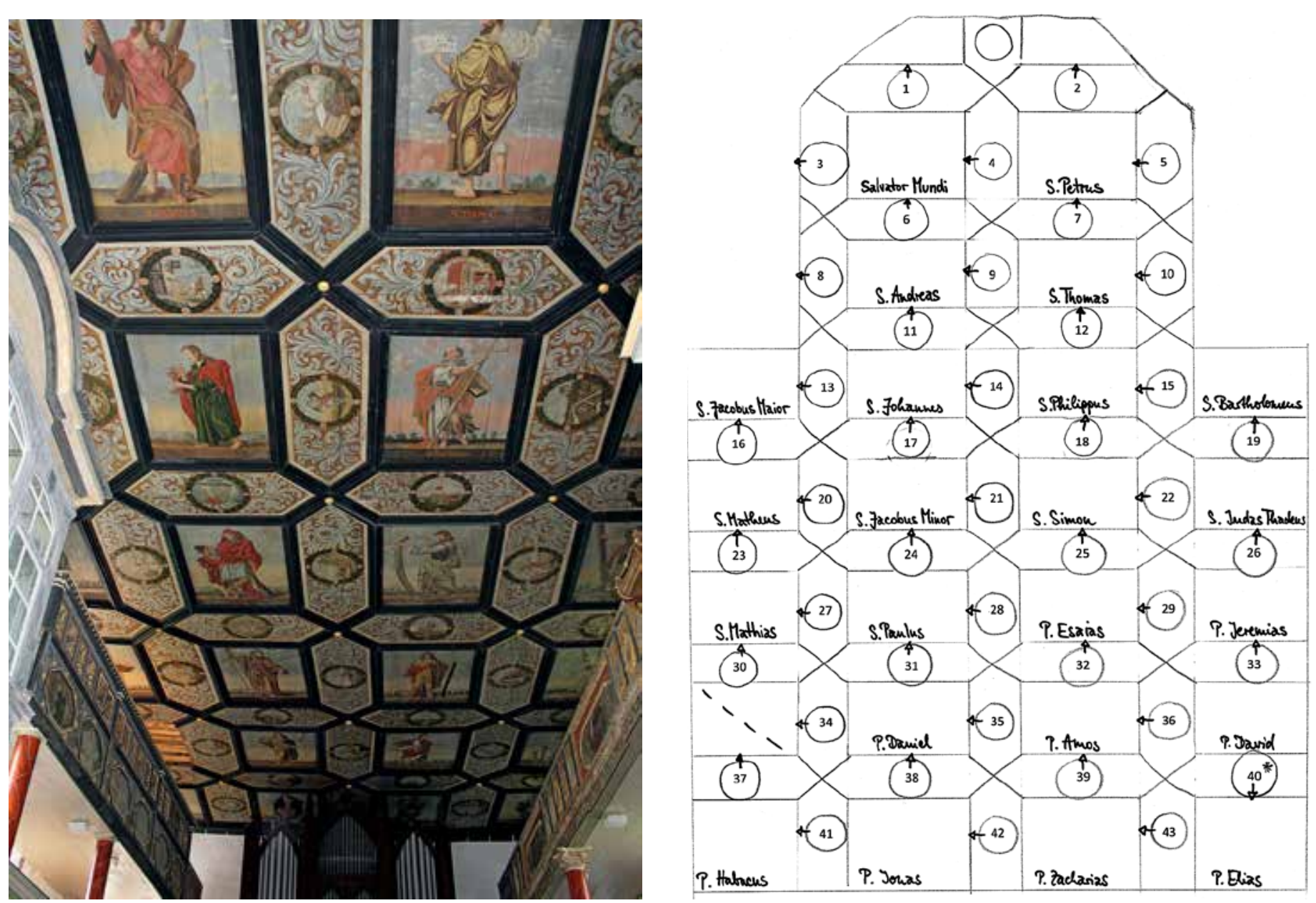

Links: St. Gotthard in Jahna, Bilderdecke

Foto: Matthias Donath

Rechts: St. Gotthard in Jahna, schematische Darstellung der Deckenfelder (Untersicht) Skizze: Susanne Magister

Zuordnung der Embleme in Jahna (vgl. Lageskizze) zu den Sonn- und Feiertagen und Nr. des Emblems in Johann Manichs „Emblemata Sacra“

1: 1. Advent, Nr. 57; 2: 2. Advent, Nr. 58; 3: 3. Advent, Nr. 60; 4: 4. Advent, Nr. 61; 5: Weihnachten, Nr. 63; 6: Sonntag nach Weihnachten, Nr. 65; 7: Stephani, Nr. 64; 8: Cantate, Nr. 16; 9: Ostern, Nr. 11; 10: Pfingsten, Nr.21, 11: 2. Sonntag nach Epiphanias, Nr. 70; 12: Heilige Drei Könige, Nr. 68; 13: Beschneidung, Nr. 66; 14: Sonntag nach Beschneidung, Nr. 67; 15: 1. Sonntag nach Epiphanias, Nr. 69; 16: 3. Sonntag nach Epiphanias, Nr. 71; 17: 4. Sonntag nach
Neuzeit großer Beliebtheit. Das in der Regel dreiteilige Emblem besteht aus einem kurzen Sinnspruch (Motto, inscriptio), einer bildlichen Darstellung (icon, pictura) und einem zusätzlichen, meist gereimten Text (epigramma, Subscriptio), wobei „die drei Elemente in ein variables und spannungsvolles Verhältnis von Darstellung und Deutung, von Verschlüsselung und Auslegung, von Sinnlichkeit und Rationalität, von Unterhaltung und Belehrung traten“2 Ein Emblem musste nicht zwingend alle Elemente enthalten.

Die Bilderdecke in der Kirche in Jahna umfasst 44 Medaillons. Das Medaillon im Scheitel des Altarraums zeigt Gottvater in der Gestalt eines Bischofs. Die anderen 43 Felder enthalten Embleme. In der schematischen Darstellung der Decke (Abbildung der Felderdecke in Untersicht) wurden sie von 1 bis 43 gezählt.

Diese Embleme sind einem Nürnberger Emblembuch entnommen, das der lutherische Pfarrer Johann Mannich (1580-1637) im Dezember 1625 unter dem Titel „Sacra Emblemata XXVI in quibus summa uniuscuiusque evangelii rotunde adumbratur, das ist sechsundsibentzig christliche Figürlein, in welche eines jeden Evangelii Summa kürtzlichen wird abgebildet" veröffentlichte. Auf dem Buchtitel ist das Jahr 1624 ange- geben. Mannich war Diakon an der Spitalkirche zum Heiligen Geist und Prediger zu St. Walburg in Nürnberg. Die Reichsstadt war in den 1520er Jahren zur Reformation übergegangen und seitdem eine Hochburg der evangelisch-lutherischen Kirche. Mannich gliederte seine Emblemsammlung nach dem Kirchenjahr. Zu jedem Sonn-, Fest- und Feiertag ist die Perikope, der gottesdienstliche Predigttext, angegeben. Dazu wird ein rundes Emblem angeboten, das sich jedoch nicht auf die Perikope, sondern auf einen eigens ausgewählten Bibelvers bezieht. Dieser steht in der kreisrunden Umschrift immer unter dem Bild. Ringsum ist weiterhin das lateinische Motto zu lesen. Unter dem Emblem befinden sich jeweils zwei lateinische Distichen, die als Subscriptio nähere Erläuterungen enthalten. Während das Emblem immer auf dem rechten Blatt der Doppelseite steht, befinden sich auf dem linken Blatt immer eine deutsche Fassung des Mottos (zwei Zeilen, gereimt) sowie eine ausführliche gereimte Auslegung. Die Embleme zeichnete der Nürnberger Maler Michael Herr (1591-1661), in Kupfer gestochen wurden sie von Peter Isselburg (1658-1630) aus Bamberg. Während die lateinischen Distichen von Christoph Höflich (1589-1630) verfasst wurden, 
stammen die deutschen Gedichte unter anderem von Daniel Schwenter, Christoph Girsner, Melchior Rinder, Christoph Höflich, Hieronymus Ammon und Paulus Nigrinus.

Während das Kirchenjahr nach lutherischer wie römisch-katholischer Tradition am 1. Adventssonntag beginnt, stehen bei Mannich die Sonntage der Fastenzeit vor Ostern an erster Stelle. Die Abfolge beginnt mit dem Sonntag Invocavit (Nr. 1) und endet mit dem Sonntag Quinquagesimae (Nr. 77). Beigefügt sind außerdem noch neun Embleme für die Stände der christlichen Gesellschaft.

Die Embleme an der Kirchendecke in Jahna folgen dem Emblembuch „Emblemata Sacra“ von Johann Mannich, geben die Inhalte des Buches aber nur in einer Auswahl wieder. Die Beschriftung enthält den Namen des Sonn- oder Feiertags in lateinischer Fassung sowie die Bibelstelle, auf die sich die Pictura bezieht. Das Motto, der eigentliche Schlüssel zum Verständnis des Emblems, fehlt. Man findet an der Decke weder das lateinische noch das deutsche Motto der Nürnberger Vorlage. Mit der Bezeichnung des Sonn- oder Feiertags allein lässt sich der Inhalt nicht erschließen.

Die Abfolge der Embleme in Jahna entspricht nicht der Abfolge in Mannichs Emblembuch. Die Reihe beginnt im Altarraum mit den Sonntagen des Advents und den Sonn- und Feierta- gen des Weihnachtsfestkreises. Anders als bei Mannich wurde demzufolge die Reihenfolge des Kirchenjahres beachtet. Integriert sind außerhalb der Reihenfolge des Kirchenjahres Ostern, Pfingsten und der Sonntag Cantate. Es folgen die Sonntage nach Epiphanias sowie die Sonntage der Vorpassionszeit. Es schließen sich die Fastensonntage vor Ostern an, bevor dann das Trinitatisfest und die Sonntage nach Trinitatis folgen. Immer wieder findet man Embleme, die aus der Reihenfolge des Kirchenjahres herausfallen. Am Ende stehen Embleme, die nicht zur Emblemfolge innerhalb der Trinitatissonntage passen: Mariä Lichtmess, Quasimodogeniti und der 5. Sonntag nach Epiphanias.

Noch einmal ist zu bemerken, dass die Embleme ihrem Inhalt nach größtenteils weder etwas mit den genannten Sonn- und Feiertagen noch mit den Perikopen zu tun haben. Auch beziehen sich die Embleme nicht auf die nebenstehenden Apostel und Propheten. Hinsichtlich der Blickrichtung wurde eine pragmatische Regelung gefunden. Die Embleme unter den großen Bildfeldern sind so ausgerichtet, dass man sie in Blickrichtung Altar lesen könnte, wenn denn der Abstand vom Boden bis zur Kirchendecke nicht so groß wäre. Die anderen Embleme links und rechts der großen Bildfelder sind in der Leserichtung von rechts nach links angeordnet.
Epiphanias, Nr. 72; 18: Quinquagesimae, Nr. 77; 19: Sexagesimae, Nr. 76; 20: Palmarum, Nr. 8; 21: Verkündigung, Nr. 7; 22: Septuagesimae, Nr. 75; 23: Invocavit, Nr. 1; 24: Reminiscere, Nr. 2; 25: 1 . Sonntag nach Trinitatis, Nr. 24; 26: Laetare, Nr. 5; 27: Judica, Nr. 6; 28: 2. Sonntag nach Trinitatis, Nr. 25; 29: Trinitatis, Nr. 23; 30: Oculi, Nr. 4; 31: 5. Sonntag nach Trinitatis, Nr. 29; 32: 6. Sonntag nach Trinitatis, Nr. 31; 33: 9. Sonntag nach Trinitatis, Nr. 37; 34: 3. Sonntag nach Trinitatis, Nr. 26; 35: 8 Sonntag nach Trinitatis, Nr. 33 36: Sonntag nach Trinitatis, Nr. 36; 37: Vocem jucunditatis, Nr. 18; 38: 7. Sonntag nach Trinitatis, Nr. 32; 39: 9. Sonntag nach Trinitatis, Nr. 32; 40: 12. Sonntag nach Trinitatis, Nr. 38; 41: Mariä Lichtmess, Nr. 73; 42: Quasimodogeniti, Nr. 13; 43: 5. Sonntag nach Epiphanias, Nr. 74

Links: St. Gotthard in Jahna, Bilderdecke, Emblem 1 Foto: Matthias Donath

Rechts: Johann Mannichs "Sacra Emblemata" (c) Archiv ZKG
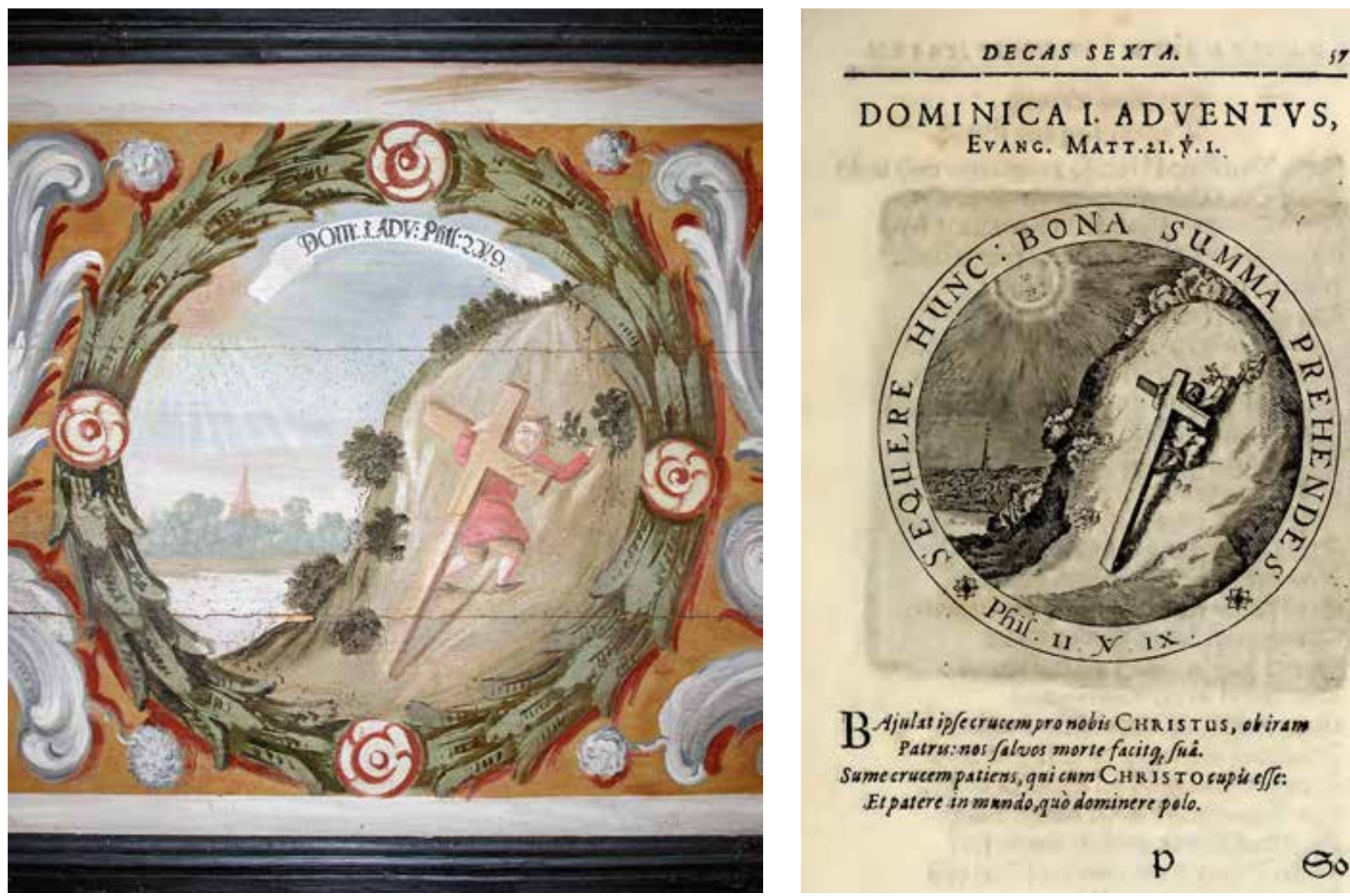

B Ajalat igfecrucempronobis Cunistus, ohiram Patru: nos falvos morle facitg, fis.

Samecrucem patiens, gai cum $\mathrm{CHR}_{\mathrm{H}}$ is T O cappis effe: Etpatere in mondo,quò dominere polo. 


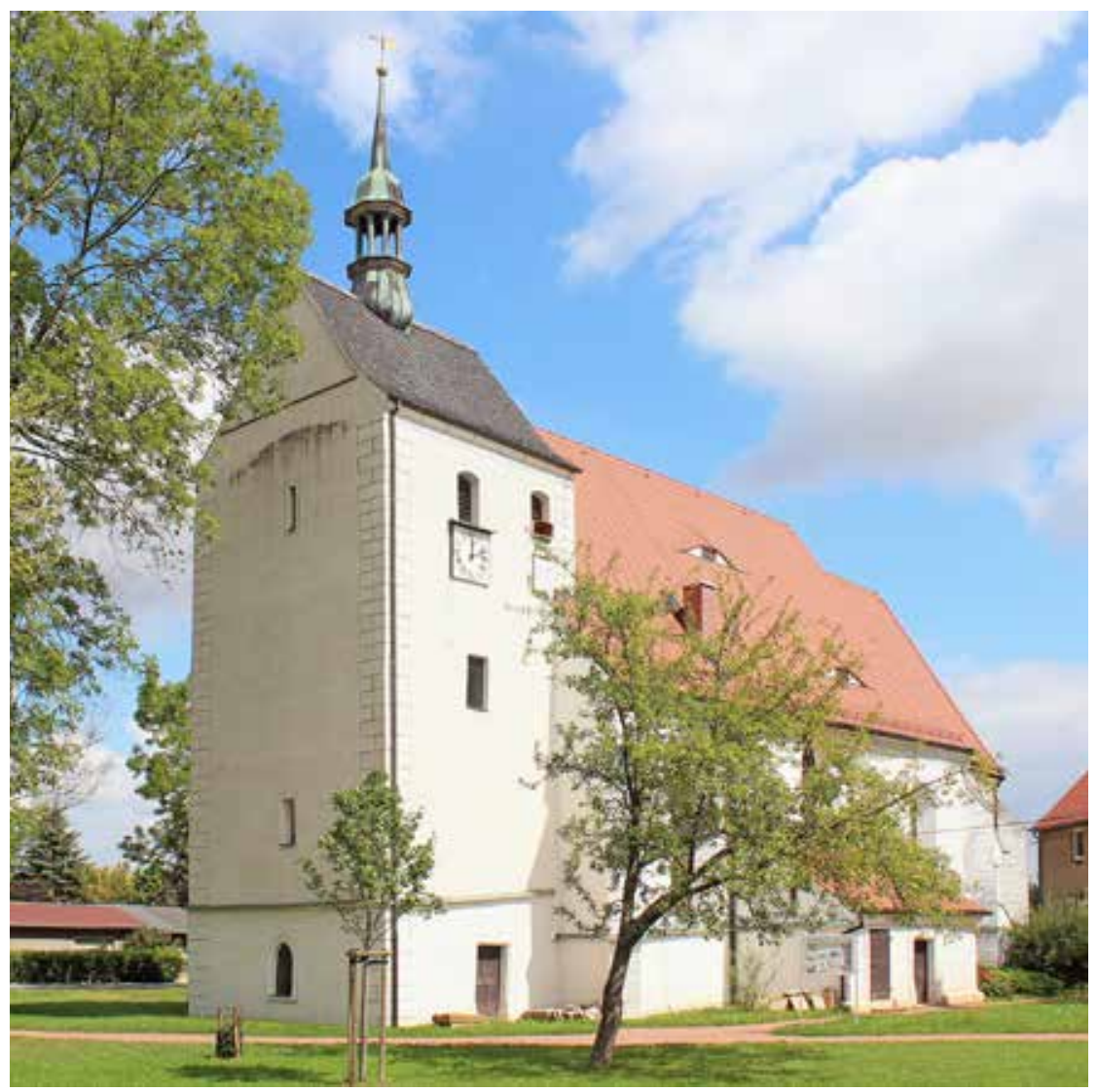

St. Gotthard in Jahna, Außenansicht Foto: Matthias Donath

\section{Autor}

Dr. Matthias Donath Herausgeber der „Sächsischen Heimatblätter“ besagt, dass ein Mensch, der die Erlösung erreichen möchte, wie Jesus Christus die schwere Last des Kreuzes, gemeint sind Leiden und Erniedrigung, auf sich nehmen muss. Ein inhaltlicher Bezug zum 1. Advent oder zur Perikope des 1. Adventsonntags (Einzug in Jerusalem) besteht nicht.

An diesem Beispiel lässt sich zeigen, dass die Embleme in Jahna nur von Betrachtern gelesen werden konnten, die über die Vorlage, Mannichs „Emblemata Sacra“, verfügten. Auch ein theologisch gebildeter Kirchenbesucher konnte die Bilder nicht so leicht erschließen. Das Bildprogramm wird sich daher kaum an die „einfachen“ Gemeindeglieder gerichtet haben, sondern an eine gebildete Zielgruppe, wohl den Auftraggeber und sein Umfeld.

Auftraggeber der Bilderdecke war vermutlich Herrmann von Wolffersdorff (1630-1703), ein Günstling des Kurfürsten Johann Georgs II. von Sachsen (1613-1680). Wolffersdorff, der erst Oberkämmerer und dann Oberhofmarschall des Kurfürsten war, kaufte 1667 das Amt Mügeln, das zum Gebiet des Hochstifts Meißen gehörte. Damit war er auch Kollator (Kirchenpatron) der Kirche in Jahna. Die Beziehung des Herrmann von Wolffersdorff zum Dresdner Hof könnte auch erklären, dass der Dresdner Maler Johann Simon Lucas den Auftrag zur Gestaltung der Felderdecke erhielt. Er war der Bruder des Dresdner Malers Gottfried Lucas, der 1671 bis 1673 die Kirche in Dresden-Leubnitz-Neuostra ausmalte. Leben und Wirken der Künstlerfamilie Lucas sind bisher noch nicht erforscht worden.

Die wissenschaftliche Bearbeitung der Bilderdecke in Jahna steht erst am Anfang. Noch fehlt eine kommentierte Darstellung der 43 Embleme mit einer theologischen Deutung und einer Einordnung in die Bild- und Texttradition der Emblematik. Außerdem sind die künstlerischen Mittel sowie die Hintergründe zu Maler und Auftraggeber genauer zu erforschen.

Schon jetzt lässt sich feststellen: Die Felderdecke der Kirche in Jahna ist in mehrfacher Hinsicht von herausragender theologischer wie kulturgeschichtlicher Bedeutung. Es handelt sich um das größte bisher bekannte emblematische Bildprogramm in einer sächsischen Kirche. Mit dem Bezug zu Mannichs „Emblemata Sacra" wird deutlich, dass Embleme auch in evangelisch-lutherischen Kirchen eine Anwendung erfahren haben. Dabei erfolgte ein Transfer vom Buch in die gebaute Architektur. Die Bilderfolge in Jahna stellt das einzige bisher bekannte Beispiel für die Verwendung von Johann Mannichs Emblembuch in einem Kirchenraum dar. 